\title{
miR-200b inhibits CD133+ glioma cells by targeting the AKT pathway
}

\author{
AIQUN LIU ${ }^{1,2}$, QINGYUN YU ${ }^{2}$, ZHONGXING PENG $^{2}$, YEQING HUANG ${ }^{2}$, \\ SHENGPENG DIAO $^{2}$, JING CHENG $^{2}$, WENTAO WANG ${ }^{3}$ and MINGFAN HONG ${ }^{2}$ \\ ${ }^{1}$ Department of Neurology, Jinan University, Guangzhou, Guangdong, 510632; \\ Departments of ${ }^{2}$ Neurology and ${ }^{3}$ Neurosurgery, School of Clinical Medicine, First Affiliated Hospital \\ of Guangdong Pharmaceutical University, Guangzhou, Guangdong 510080, P.R. China
}

Received June 17, 2015; Accepted October 24, 2016

DOI: $10.3892 / 01.2017 .6055$

\begin{abstract}
MicroRNA-200b (miR-200b) is a tumor suppressor in multiple tumor types, including gastric cancer, breast cancer, ovarian cancer and glioma. The biological significance of a known normal and cancer stem cell marker, CD133, remains elusive. The aim of the present study was to identify the function and mechinism of miR-200b in suppressing CD133+ glioma cells. CD $133^{+}$glioma cells were sorted by flow cytometry. The expression of miR-200b, Ki67, GAP43, GFAP and CD133 were tested by reverse transcription-quantitative polymerase chain reaction. The binding of $\mathrm{miR}-200 \mathrm{~b}$ to prominin 1 (PROM1) was certificated by luciferase reporter assay. Cell proliferation was analyzed by bromodeoxyuridine staining. The protein level of CD133, p-AKT, AKT and Notch1 was detected by western blot analysis. Analysis of glioma samples revealed that CD133 expression is negatively associated with miR-200b. PROM1, which is the gene that codes CD133, was certified to be a target of miR-200b. miR-200b expression inhibited the stemness properties and division of the $\mathrm{CD} 133^{+}$glioma cells. Our results identified a miR-200b/CD133/PI3K/Akt signaling axis, exploring the fundamental role of miR-200b and CD133 in glioma stem cell behavior.
\end{abstract}

\section{Introduction}

Glioma is one of the most common primary central nervous system tumors with high mortality and poor 5-year survival rate (1). Gliomas represent a disparate group of tumors for which there are to date no cure. Thus, there is an urgent need for novel diagnostic and therapeutic methods based on the increased understanding of the molecular mechanisms

Correspondence to: Dr Mingfan Hong, Department of Neurology, School of Clinical Medicine, First Affiliated Hospital of Guangdong Pharmaceutical University, 16 Jichang Load, Guangzhou, Guangdong 510080, P.R. China

E-mail: hongmingfan9001@126.com

Key words: glioma, miR-200b, CD133, Akt of glioma (2). Cancer stem-like cells (CSCs) are poorly differentiated multipotent tumor-propagating cells that disproportionately contribute to therapeutic resistance and tumor recurrence (3). Research has been performed to identify the approaches to inhibit CSCs. Suppression of stathmin, an oncogene, inhibited invasion and enhanced chemotherapy sensitivity of stem cells derived from glioma cell lines (4).

AKT serine-threonine kinase 1 (AKT) is a downstream target and effector of phosphatidylinositol 3-kinase (PI3K). AKT is considered to be a key regulator of cell growth and fate decisions in tumors (5). It has been reported that inhibition of the AKT pathway could suppress glioma (6). CD133 is a marker of glioma stem-like cells. A previous study demonstrated that the CSC marker, CD133, was associated with activated AKT and radiation resistance in colon cancer cells (7). Zhu et al (8) identified that overexpression of CD133 enhanced chemoresistance to 5-fluorouracil by activating the PI3K/AKT/ribosomal protein S6 kinase pathway in gastric cancer cells.

MicroRNAs (miRNAs) are small non-coding RNAs which have an important role in regulating gene expression (9). MicroRNA-200b (miR-200b) targets protein kinase $\mathrm{C} \alpha$ and suppresses triple-negative breast cancer metastasis (10). The restoration of miR-200b expression may inhibit the maintenance of CSC properties and the reverse chemoresistance of CSCs. The histone deacetylase 1/miR-200b/Suz-12-E-cadherin signaling may account for maintenance of CSCs (11). The current study aimed to identify the role of miR-200b in $\mathrm{CD}_{133}{ }^{+}$glioma cells.

\section{Materials and methods}

Cell culture and sorting. Cells (TC1 and TC2 cells) were isolated from primary surgical glioma biopsy specimens in accordance with protocols as previously described (12). The U251 glioma cell line was purchased from the Chinese Academy Medical Science (Beijing, China). The cells were cultured in RPMI-1640 medium supplemented with $10 \%$ fetal bovine serum (FBS), penicillin and streptomycin, all of which were obtained from Gibco (Thermo Fisher Scientific, Inc., Waltham, MA, USA) and were cultured in a humidified chamber with $5 \% \mathrm{CO}_{2}$ at $37^{\circ} \mathrm{C}$. All transfections were performed using Lipofectamine 2000 (Invitrogen; Thermo 
Fisher Scientific, Inc.). Magnetic beads (Dynabeads M-450 Epoxy; Invitrogen; Thermo Fisher Scientific, Inc.) labeled with CD133 antibody (catalog no., PA5-38014; Thermo Fisher Scientific, Inc.) were incubated with $2.5 \times 10^{6}$ cells in $1 \mathrm{ml} \mathrm{B1}$ solution containing Dynabeads for $30 \mathrm{~min}$ at $4^{\circ} \mathrm{C}$. In total, $25 \mu \mathrm{l}$ of labeled Dynabeads was incubated with $2.5 \times 10^{6}$ cells Suspension was incubated for $20 \mathrm{~min}$ at $4^{\circ} \mathrm{C}$ with gentle rotation followed by 2 min positive isolation using an EasySep magnet (cat. no. 18000; Stemcell Technologies, Inc., Beijing, China). Bead-bound cells were washed 4 times using $1 \mathrm{ml}$ PBS buffer.

Tissue samples. Glioma samples $(\mathrm{n}=80)$ and normal brain tissues $(n=20)$ were collected at the The First Affiliated Hospital of Guangdong Pharmaceutical University (Guangzhou, China) with written consent from patients or family members of patients in accordance with institutional guidelines as approved by the First Affiliated Hospital of Guangdong Pharmaceutical University. Pathology was graded according to World Health Organization criteria. The RNA were extracted from the tissues using TRIzol reagent (Thermo Fisher Scientific, Inc.) for reverse transcription-quantitative polymerase chain reaction (RT-qPCR) detection.

$R T-q P C R$. RT of specific miRNAs (from $10 \mathrm{ng}$ of total RNA) was performed using the real-time loop primers for each type of miRNA and the TaqMan miRNA RT kit from Applied Biosystems (Thermo Fisher Scientific, Inc., Waltham, MA, USA). cDNA obtained using GoScript ${ }^{\mathrm{TM}}$ Reversion Transcription Mixes (Promega Corporation, Madison, WI, USA) was used for quantitative TaqMan PCR using the real-time primers provided, according to the manufacturer's instructions. $\mathrm{Cq}$ values were converted to fold expression changes (2- $2^{-\Delta \mathrm{Cq}}$ values) (13) following normalization to U6 small nuclear RNA (primers; forward 5'-CTCGCTTCGGCAGCA CA-3', reverse 5'-AACGCTTCACGAATTTGCGT-3'). For mRNA analysis, miR-200b mimics, inhibitor sequences or control sequences were transfected into glioma cells. The primer sequences were as follows: hsa-miR-200b mimics, 5'-CAUCUUACUGGGCAGCAUUGGA-3'; miR-NC, 5'-UUC UCCGAACGUGUCACGUTT-3'; miR-200b inhibitor, 5'-UCAUCAUUACCAGGCAGUAUUA-3'; inh-NC, 5'-CAG UACUUUUGUGUAGUACAA-3' (GenePharma, Shanghai, China). RT was performed on total RNA using random primers (GE Healthcare Life Sciences, Chalfont, UK), and GAPDH (forward 5'-TTGCCATCAATGACCCCTTCA-3', reverse 5'-CGCCCCACTTGATTTTGGA-3') were used to control for cDNA concentration in a separate $\mathrm{PCR}$ reactions for each sample. LightCycler Fast Start DNA Master SYBR Green Mix (Roche Diagnostics GmbH, Mannheim, Germany) was added to each PCR reaction along with cDNA and 1 pmol primer in a total volume of $10 \mu \mathrm{l}$.

Luciferase assays. The full-length of the 3' untranslated regions (UTRs) of the prominin 1 (PROM1) gene, which encodes CD133, was amplified by PCR from U251 cells genomic DNA and inserted into the pGL3 control vector (Promega Corporation) using the XbaI site immediately downstream from the stop codon of luciferase. Several inserts with deletions of $6 \mathrm{bp}$ from the site of perfect complementarity of the PROM1 gene were generated using the Qiagen XL-Site Directed Mutagenesis kit (Qiagen, Inc., Valencia, CA, USA). U251 cells were cotransfected using nucleoporation (Amaxa; Lonza Group, Ltd., Basel, Switzerland) according to the manufacturer's protocol (solution $\mathrm{V}$, programme T-016) using $5 \mu \mathrm{g}$ of the firefly luciferase reporter vector and $0.5 \mu \mathrm{g}$ of the control vector containing Renilla luciferase (pRL-TK; Promega Corporation). Each nucleoporation used $50 \mathrm{nM}$ of the miR-200b or a scrambled oligonucleotide. Firefly and Renilla luciferase activities were measured consecutively using the dual luciferase assay and Thermo Scientific Multiskan MK3 Microplate Reader (Thermo Fisher Scientific, Inc.) 48 h after transfection.

Cell number counting. A total of 10,000 TC1 and TC2 cells were plated onto a 24 -well plate. Upon attachment, cells were transfected with scramble control miRNA, miR-200b mimics, inhibitor control or miR-200b inhibitor. Each group was evaluated in duplicate in six wells. After being transfected for 24, 48, 72 or $96 \mathrm{~h}$, cells were digested with $100 \mu \mathrm{l}$ trypsin, and then $0.5 \mathrm{ml}$ RPMI-1640 medium supplemented with $10 \%$ FBS, penicillin and streptomycin was added. Upon mixing, $10 \mu \mathrm{l}$ cell suspension was added into a slide, which was then inserted into a cell counter (Bio-Rad Laboratories, Inc., Hercules, CA, USA) to count the cell number.

Bromodeoxyuridine (BrdU) cell proliferation assay. The cell proliferation assay was performed by measuring BrdU incorporation into the newly synthesized DNA of replicating cells. Cells were then infected with miRNA mimics and inhibitor. The cells were loaded with BrdU (Roche Applied Science, Penzberg, Germany) in the last $4 \mathrm{~h}$ of treatment with miRNA mimics or inhibitor. BrdU incorporation was quantified by BrdU immunohistochemical staining kit (Abcam, Cambridge, UK) following manufacturer's instructions. Three fields were chosen randomly from 10 sections to ensure objectivity of sampling. Digital images were acquired using a confocal microscope. Each assay was repeated three times. From total of 100 cells from each field the ratio of BrdU-positive cell was calculated.

Western blot. Glioma cells were transfected with CD133 siRNA (sense, 5'-GGCUGCUGUUUAUUAUUCUTT-3' and antisense, 5'-AGAAUAAUAAACAGCAGCCTT-3') and nonspecific siRNA (sense, 5'-UUCUCCGAACGUGUCACGUTT-3' and antisense, 5'-ACGUGACACGUUCGGAGAATT-3') (Riobio, Guangzhou, China). The proteins from glioma cell lines were extracted using RIPA lysis buffer with a proteinase inhibitor. The protein concentration in the lysates was measured with the Protein Bicinchoninic Acid Assay kit (Bio-Rad Laboratories, Inc.), and $20 \mu \mathrm{g}$ of the total protein mixed with $2 \mathrm{X}$ SDS loading buffer was loaded per lane. The proteins in the lysates were separated by $12 \%$ SDS-PAGE and transferred to polyvinylidene difluoride (PVDF) membranes (EMD Millipore, Billerica, MA, USA). To block nonspecific binding, the membranes were incubated at room temperature for $1 \mathrm{~h}$ with $5 \%$ skim milk powder. The PVDF membranes were then incubated for $12 \mathrm{~h}$ at $4^{\circ} \mathrm{C}$ with an antiserum containing antibodies against CD133 rabbit monoclonal antibody $(\mathrm{mAb})$ (dilution, 1:1,000; catalog no., 5860; Cell Signaling Technology, Inc., Danvers. MA, USA), 
A

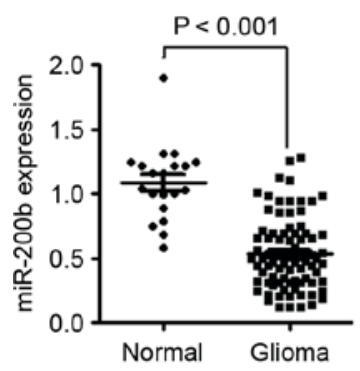

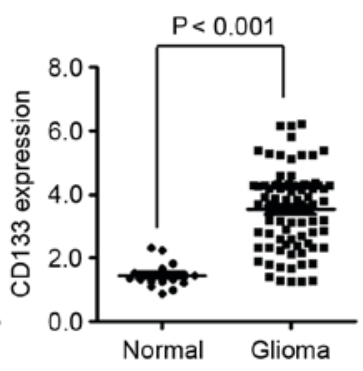

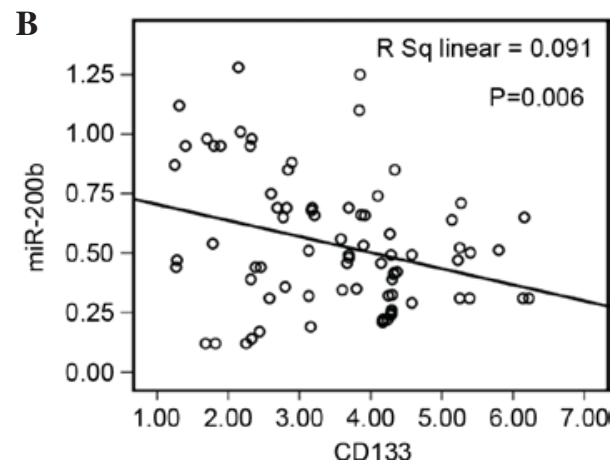

Figure 1. miR-200b suppresses the expression of CD133 in glioma cells. (A) The expression of miR-200b and CD133 in 20 normal brain tissues and 80 glioma samples. (B) Correlation between the expression of miR-200b and CD133 in glioma tissues. miR, microRNA.

A

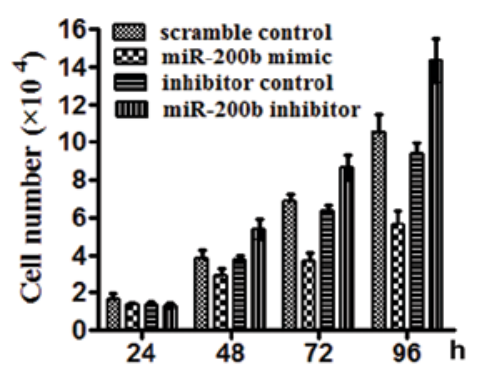

TC1

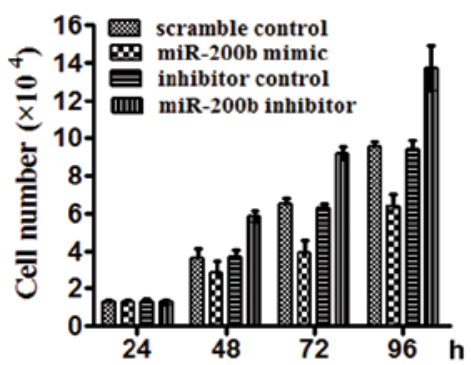

TC2

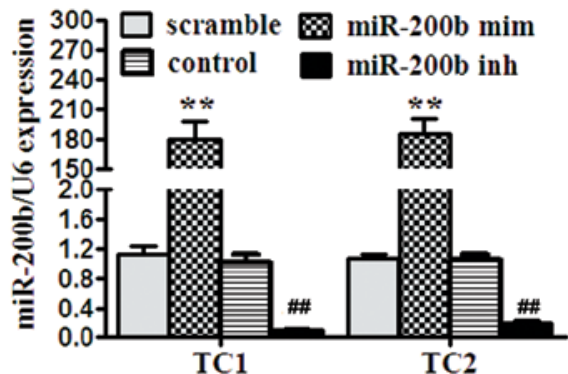

miR-200b inhibitor

inhibitor control

B
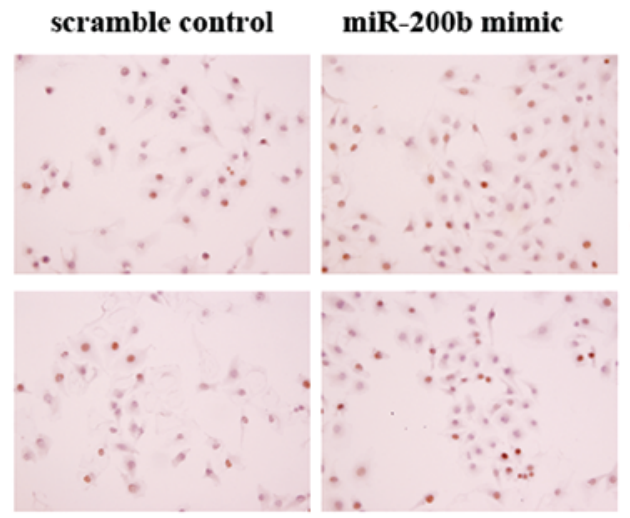

C

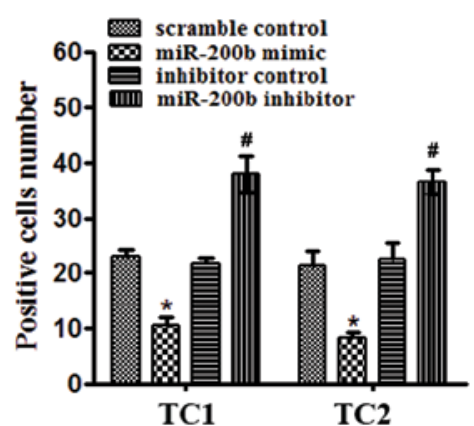

TC1

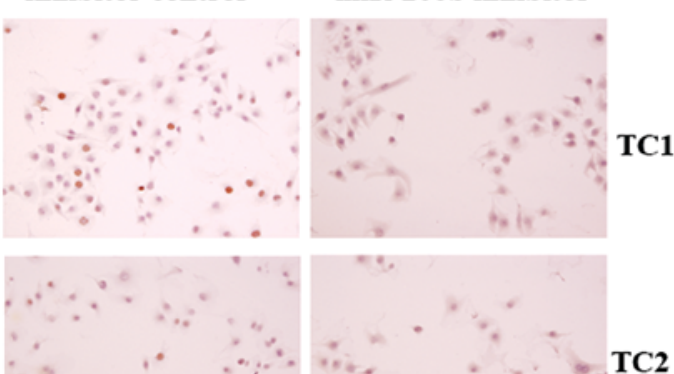

TC2

Figure 2. miR-200b levels are essential for proliferation and stem-like properties of human glioma cells. (A) Left and middle: Number of TC1 and TC2 cells infected with scrambled control, miR-200b mimics or miR-200b inhibitor; Right: The miR-200b expression was detected by RT-qPCR. (B) Imaging of TC1 and TC2 cells cells positive for BrdU staining. (C) Quantification of TC1 and TC2 cells positive for BrdU staining. (D) RT-qPCR detected Ki67, GAP43, GFAP and CD133 levels in TC1 cells infected with scrambled control, miR-200b mimics or miR-200b inhibitor. ${ }^{*} \mathrm{P}<0.05,{ }^{* *} \mathrm{P}<0.01$ vs. scramble; ${ }^{\# \mathrm{P}}<0.05$, ${ }^{\text {\#" }} \mathrm{P}<0.01$ vs. inhibitor control. miR, microRNA; GAP43, growth-associated protein 43; GFAP, glial fibrillary acidic protein.

GFAP mouse mAb (dilution, 1:200; catalog no., sc-71143; Santa Cruz Biotechnology, Inc., Dallas, TX, USA), p-AKT rabbit mAb (dilution, 1:1,000; catalog no., 4060; Cell Signaling Technology,
Inc.), AKT rabbit mAb (dilution, 1:1,000; catalog no., 4691; Cell Signaling Technology, Inc.), Notch1 rabbit mAb (dilution, 1:1,000; catalog no., 3608; Cell Signaling Technology, Inc.) and 

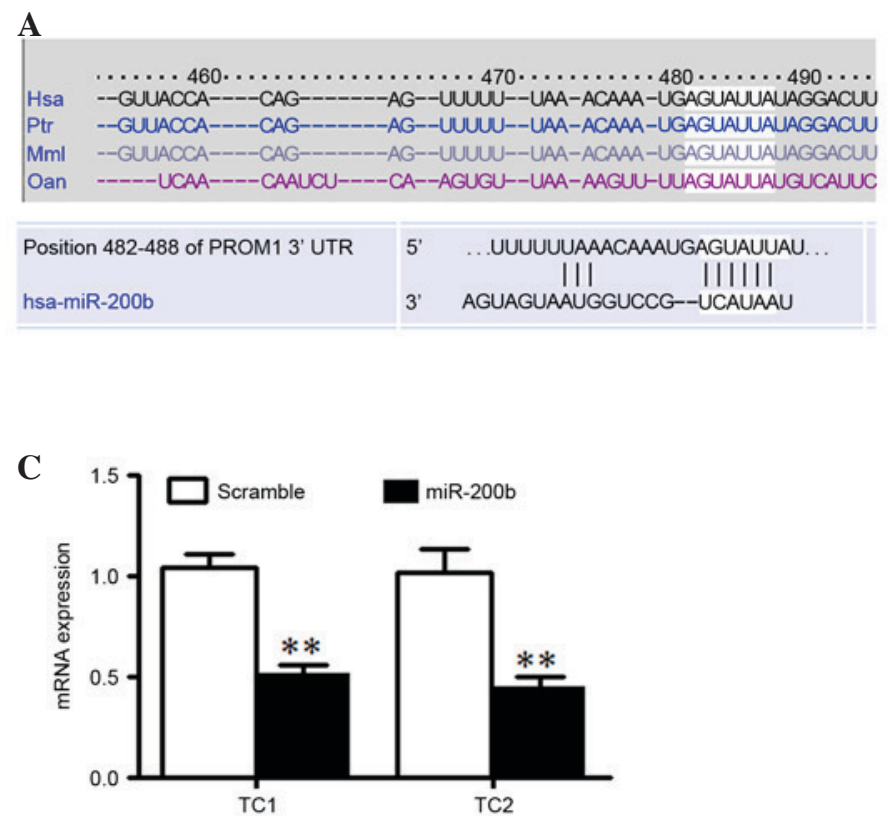

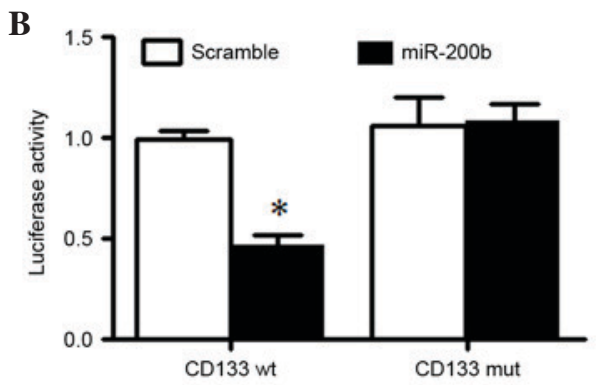

D

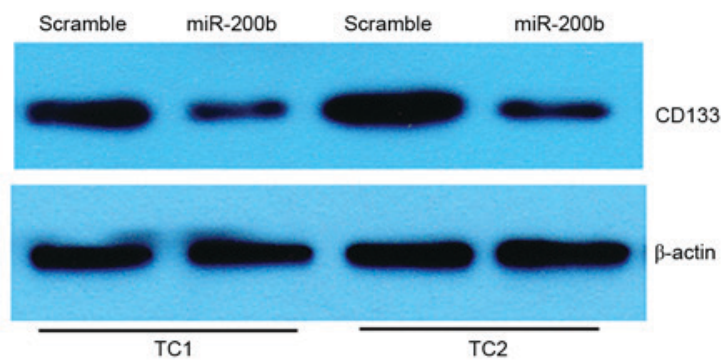

Figure 3. PROM1 is a direct target of miR-200b. (A) Schematic representation of putative miR-200b-binding sites in the 3'UTR of PROM1. (B) U251 cells were cotransfected with wt or mut PROM1 3'-UTR, pGL-3 control and either miR-200b or negative control. Relative firefly luciferase activity was normalized to Renilla luciferase activity. (C) Expression of PROM1 mRNA was detected by reverse transcription-quantitative polymerase chain reaction in TC1 and TC2 cells transfected with miR-200b mimics or control mimic and in these two cells transfected miR-200b inhibitor or control inhibitor. (D) Protein level of CD133 was detected by western blot analysis in TC1 and TC2 cells transfected with miR-200b mimics or control mimic and in these cells transfected with miR-200b inhibitor or control inhibitor. GAPDH was used as an internal control. ${ }^{~} \mathrm{P}<0.05,{ }^{* *} \mathrm{P}<0.01$ vs. scramble control. $3^{\prime} \mathrm{UTR}, 3^{\prime}$ untranslated region; miR, microRNA; PROM1, prominin 1.

$\beta$-actin mouse mAb (dilution, 1:1,000; catalog no., 3700; Cell Signaling Technology, Inc.). Secondary antibodies consisted of rabbit anti-mouse horseradish perosidase (HRP)-conjugated (catalog no., BA1058) and goat anti-rabbit HRP-conjugated (catalog no., BA1058) obtained from Boster (Wuhan, China). The secondary antibodies were diluted at 1:5,000 and incubated with the membrane at room temperature for $1 \mathrm{~h}$ and enhanced chemiluminescence western blot detection reagents (New England Biolabs, Inc., Ipswich, MA, USA) were used to visualize the target proteins, which were quantified with a Bio Image Intelligent Quantifier 1-D (version 2.2.1; Nikon, Tokyo, Japan).

Statistical analysis. In general, significance was analyzed by unpaired two-tailed Student $t$ test using GraphPad InStat 5.0 software (GraphPad Software, Inc., La Jolla, CA, USA). The significance of CD133 expression differences in glioma samples was determined by using Student $t$ test (two-tailed). Results are expressed as the mean \pm standard deviation. Pearson correlation assay was used to analyze the correlation between CD133 expression and miR-200b expression. $\mathrm{P}<0.05$ was considered to indicate a statistically significant difference.

\section{Results}

CD133 expression is elevated in gliomas and negatively correlated with the expression of miR-200b. Analysis of the expression of miR-200b and mRNA levels of CD133 in glioma tissues was conducted using tissues obtained from the First Affiliated Hospital of Guangdong Pharmaceutical University. The expression of miR-200b was significantly downregulated
$(\mathrm{P}<0.01)$ and $\mathrm{CD133}$ was significantly upregulated $(\mathrm{P}<0.01)$ in glioma compared with normal tissues (Fig. 1A). Statistical analysis indicated that the expression of $\mathrm{CD} 133$ was negatively correlated with miR-200b in glioma tissues ( $\mathrm{P}=0.06$; Fig. 1B).

miR-200b levels are required for proliferation and stem-like properties of human glioma cells. To address the importance of miR-200b in gliomas in vitro, the TC1 and TC2 glioma tissues initiated cell lines were used. Cells were transfected with miR-200b mimics, a scrambled control, miR-200b inhibitor or a negative control and were subsequently collected. Cell number counting assay demonstrated that miR-200b reduced cell growth compared with the scramble control and inhibition of miR-200b increased cell number compared with the negative control after culture for 48 and $72 \mathrm{~h}$ in TC1 and TC2 cells (Fig. 2A). In TC1 and TC2 cells, the effect of miR-200b on proliferation was confirmed by BrdU staining (Fig. 2B and C). The number of BrdU-positive TC1 and TC2 cells was decreased by miR-200b mimcs compared with scramble control $(\mathrm{P}<0.05)$, but increased by miR-200b inhibitor compared with the inhibitor control $(\mathrm{P}<0.05)$, as detected by BrdU staining (Fig. 2C). miR-200b in TC1 cells significantly reduced mRNA expression of the proliferation marker, Ki67 $(\mathrm{P}<0.05)$, and GAP43 $(\mathrm{P}<0.05)$, a marker of mature neurons compared with the scramble control, and also decreased the levels of markers known to identify normal stem-like cells and brain tumor-initiating cells, CD133 $(\mathrm{P}<0.05)$, and GFAP $(\mathrm{P}<0.05)$. miR-200b inhibitor had the opposite effect (Fig. 2D). These data prompted the hypothesis that miR-200b levels may be important for proliferation and directing the fate certain cells in glioma. 
A

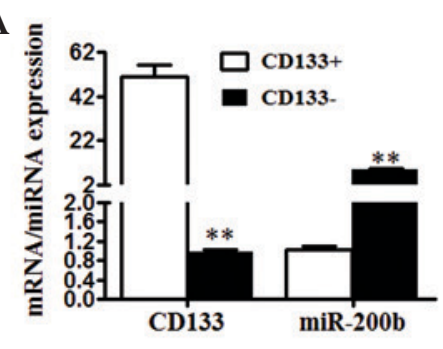

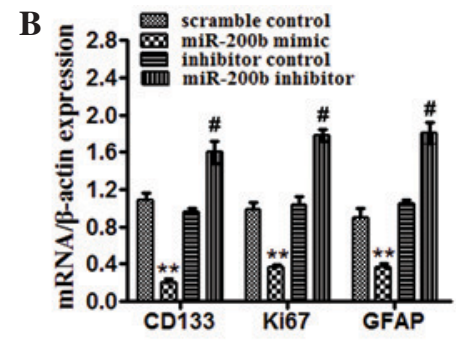

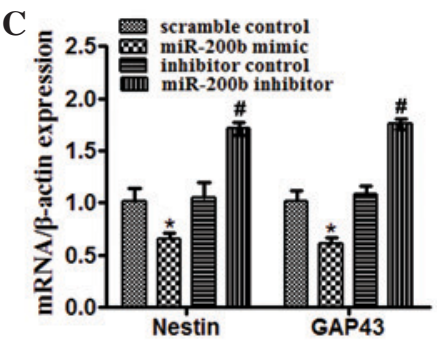

D

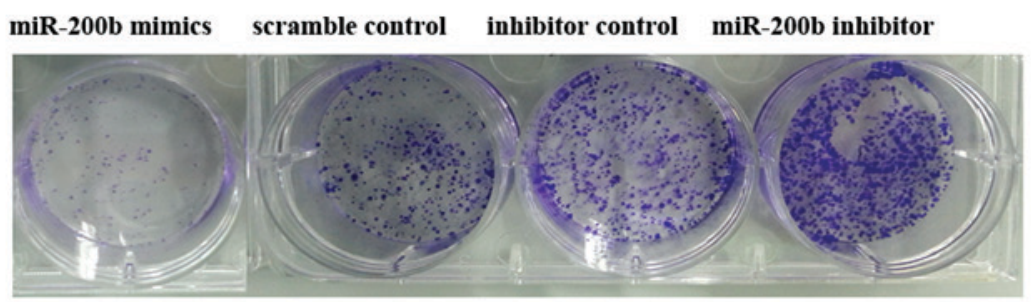

$\mathbf{E}$

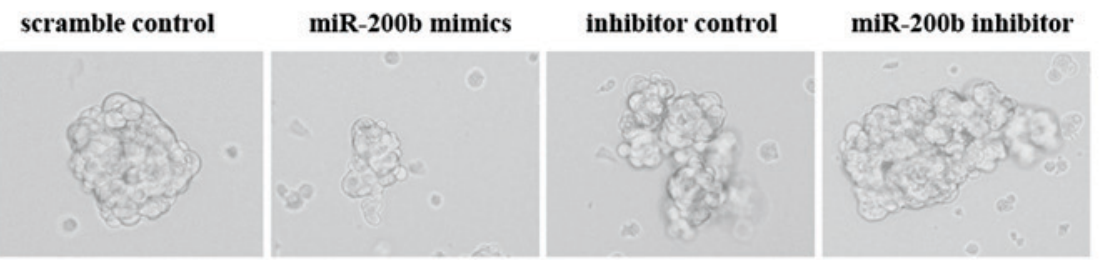

$\mathbf{F}$
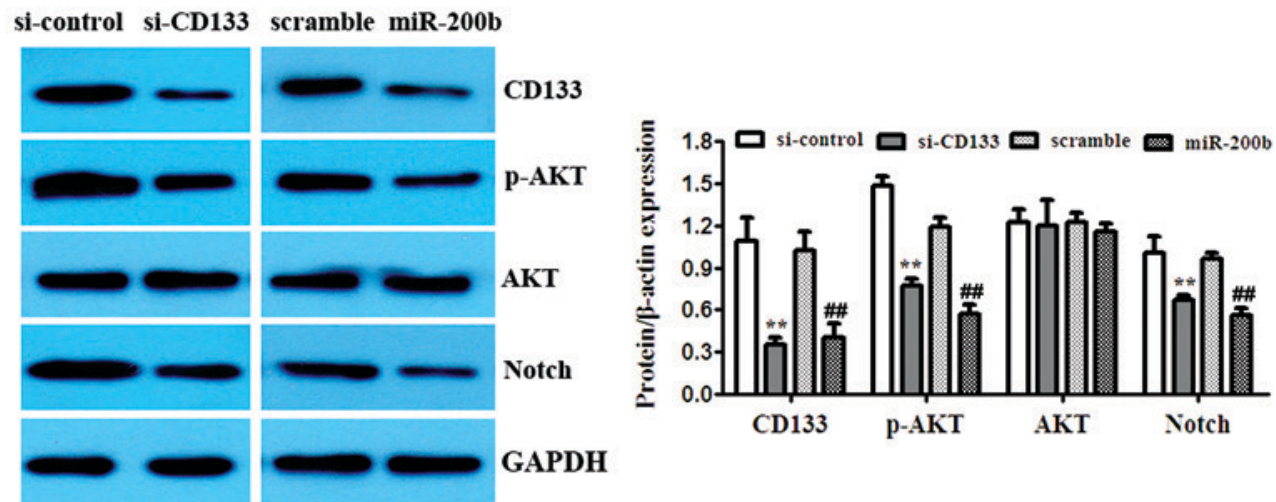

Figure 4. miR-200b expression has a critical role in the stemness properties and division of the $\mathrm{CD}_{133^{+}}$glioma cells. (A) $\mathrm{TC} 1$ cells were sorted and $\mathrm{CD} 133^{+}$ and CD133 populations were analyzed by RT-qPCR for CD133 and miR-200b levels. ${ }^{* *} \mathrm{P}<0.01$ vs. CD133 ${ }^{+}$. (B) TC1 cells were sorted for CD133. The CD133 population was transfected with scrambled control, miR-200b mimics or miR-200b inhibitor. Overexpression and knockdown confirmed by RT-qPCR. Effects on CD133, Ki67 and GFAP mRNA levels. (C) RT-qPCR of different markers (Nestin, GAP43) from the CD133+ population transfected with scrambled control, miR-200b mimics or miR-200b inhibitor. " $\mathrm{P}<0.05,{ }^{* *} \mathrm{P}<0.01$ vs. scramble control; ${ }^{\#} \mathrm{P}<0.05,{ }^{\# \#} \mathrm{P}<0.01$ vs. inhibitor control. (D) Clonal assay was performed on control, miR-200b mimics and miR-200b inhibitor transfected CD133+ $\mathrm{TC} 1$ cells. (E) Neurosphere formation was detected in CD133 ${ }^{+} \mathrm{TC} 1$ cells transfected with control, miR-200b mimics and inhibitor. (F) The activity of AKT signaling (CD133, p-AKT, total AKT, Notch) in CD133+ cells with CD133 knockdown or miR-101 overexpression tested by western blot. GAPDH was used as an internal control. ${ }^{* *} \mathrm{P}<0.01 \mathrm{vs}$. si-control; ${ }^{\# \prime} \mathrm{P}<0.01$ vs. scramble. RT-qPCR, reverse transcription-quantitative polymerase chain reaction; miR, microRNA; GFAP, glial fibrillary acidic protein; GAP43, growth-associated protein 43; si, small interfering RNA; p-, phosphorylated; AKT, AKT serine/threonine kinase 1.

PROM1 is a direct target of miR-200b. We used Targetscan (http://www.targetscan.org/mamm_31/) to help identify miR-200's targets in human glioma. PROM1 was predicted (Fig. 3A), which encodes CD133 protein. The full-length PROM1 3'-UTR was cloned downstream of the firefly luciferase gene and cotransfected with miR-200b mimics or scrambled oligonucleotides and LNA-modified anti-miR-200b oligonucleotide or a control oligonucleotide. U251 cells cotransfected with wild type PROM1 reporter constructs and
miR-200b mimics exhibited a significant reduction of luciferase activity compared with cells transfected with scramble control miRNA $(\mathrm{P}<0.05$; Fig. 3B). Additionally, mutation of the putative miR-200b target sites in the 3'-UTR of PROM1 abrogated luciferase the response to miR-200b (Fig. 3B). miR-200b mimic or a scrambled oligonucleotide was transfected into TC1 and TC2 cells. The result demonstrated that there was a marked reduction of the mRNA and protein level of CD133 in miR-200b overexpressed TC1 and TC2 cells 
compared with scrambled control (Fig. 3C and D). Taken together, these results indicated that miR-200b downregulates CD133 expression by directly targeting its $3^{\prime} \mathrm{UTR}$.

miR-200b expression has a critical role in the stemness properties and division of the CD133+ glioma cells. TC1 cells with and without CD133 expression were isolated. The expression of miR-200b in $\mathrm{CD} 133^{+} \mathrm{TC} 1$ cells was higher than in CD133- TC1 cells (Fig. 4A). To determine the potential role of miR-200b in the $\mathrm{CD} 133^{+}$glioma cells, multiple proliferation and stem-like markers were detected. Transfection of TC1 cells with miR-200b mimics significantly reduced the mRNA levels of the proliferation marker Ki67, GFAP, Nestin and GAP43 (Fig. 4B and C). The miR-200b inhibitor exerted the opposite effect (Fig. 4B and C). To determine the potential role of miR-200b in the $\mathrm{CD} 133^{+}$populations with neurosphere formation capacity, U251 cells were cultured and passaged in monolayers or as spheres to promote self renewal in vitro. Overexpression of miR-200b suppressed colony and sphere formation of $\mathrm{CD}_{133^{+}} \mathrm{TC} 1$ cells. However, miR-200b inhibitor enhanced the colony and sphere formation of $\mathrm{CD} 133^{+} \mathrm{TC} 1$ cells (Fig. 4D and E).

miR-200b regulates $A K T$ signaling by targeting CD133 in $\mathrm{CD}_{133^{+}}$glioma cells. The activity of AKT signaling was detected in $\mathrm{CD}_{133^{+}}$glioma cells treated with miR-200b mimics and CD133 was knocked down. The data demonstrated that overexpression of miR-200b and inhibition of CD133 reduced levels of phosphorylated AKT and Notch compared with the scramble miRNA and control si, respectively, but had no effect on total Akt (Fig. 4F).

\section{Discussion}

Many CSCs markers have been suggested to be potential prognostic and predictive markers in glioma, including CD133 and Nestin. CD133 and Nestin were detected in all histological subtypes of glioma, but predominantly in WHO grade III and IV tumors (14). The present study identified that CD133 was overexpressed in glioma cell lines and tissues.

miR-200b is a member of the miR-200 family that suppress tumors. The miR-200 family members are strongly associated with pathological epithelial to mesenchymal transitions (EMT) and to have a metastasis suppressive role (15). miR-200b suppresses arsenic-transformed cell migration by targeting protein kinase $\mathrm{C} \alpha$, the Wnt5b-protein kinase $\mathrm{C} \alpha$ positive feedback loop and inhibiting Racl activation (16). miR-200b was downregulated in the adriamycin-resistant small lung cancer H69AR cells and enforced expression of miR-200b by miRNA mimics increased cell sensitivity to adriamycin (17). A previous study demonstrated that miR-200b may be a novel and valuable marker for predicting the clinical outcome of patients with glioma (18). miR-200b was reported to be prognostic factor and to target multiple members of the RAB GTPase family in glioma (19). The current study identified that miR-200b was downregulated and negatively correlated with the expression of CD133 in glioma tissues.

CD133 is encoded by the PROM1 gene. Expression of PROM1 in cancer is considered similar to the expression and function observed in normal stem cells. Overexpression of
PROM1 is inversely correlated with isocitrate dehydrogenase (R132H) mutation. These findings support thay PROM1 functions as a tumor cell-intrinsic marker associated with glioma survival (20). Use of the online software, Targetscan, indicated that PROM1 is a direct target of miR-200b. The the best of our knowledge, the current study is the first to demonstrate that PROM1 is a direct target of miR-200b.

Glioma stem cells have self-renewal capability and are resistant to conventional chemotherapy. The role of miRNA dysregulation in stemness and division remains to be identified. A recent study demonstrated that targeting the miR-34a-Notch1 axis reduces breast cancer stemness and chemoresistance (21). miR-145 is a strong repressor of EMT. The transcriptional repressor zinc-finger E-box binding homeobox 2 (ZEB2) is a target of miR-145, and it can also regulate the expression of miR-145. The ZEB2/miR-145 double-negative feedback loop is important for the control of EMT and stem cell properties during the bone metastasis of prostate cancer cells (22). miR-300 promotes self-renewal and inhibits the differentiation of glioma stem-like cells (23). Although miR-200b was demonstrated to be a tumor suppressor, its role in stemness properties remains unknown. The present study initially identified that the expression of miR-200b was essential for proliferation and stem-like properties of human glioma cells, TC1 and TC2. Subsequently, $\mathrm{CD} 133^{+}$cells were isolated from the glioma cell population and revealed that miR-200b expression was important for the stemness properties and proliferation of $\mathrm{CD}_{133^{+}}$ glioma cells.

A previous study demonstrated that upregulation of miR-200b in patients with biliary atresia accelerates the proliferation and migration of hepatic stallate cells by activating PI3K/AKT signaling (24). Zinc finger protein, FOG family member 2 downregulation by transforming growth factor- $\beta 1$-induced miR-200b/c leads to AKT kinase activation and glomerular mesangial hypertrophy associated with diabetic nephropathy (25). The present study aimed to understand the association between miR-200b and AKT signaling in glioma stem-like cells. The data indicated that overexpression of miR-200b and inhibition of CD133 activated AKT signaling in $\mathrm{CD} 133^{+}$glioma cells.

\section{Acknowledgements}

This study was supported by the National Science Foundation of China (grant no. 81173418).

\section{References}

1. Chatterjee M, Li K, Chen L, Maisano X, Guo Q, Gan L and Li JY: Gbx2 regulates thalamocortical axon guidance by modifying the LIM and Robo codes. Development 139: 4633-4643, 2012.

2. Deng Y, Wang J, Wang G, Jin Y, Luo X, Xia X, Gong J and $\mathrm{Hu}$ J: p55PIK transcriptionally activated by MZF1 promotes colorectal cancer cell proliferation. Biomed Res Int 2013: 868131, 2013.

3. Deplus R, Blanchon L, Rajavelu A, Boukaba A, Defrance M, Luciani J, Rothé F, Dedeurwaerder S, Denis H, Brinkman AB, et al: Regulation of DNA methylation patterns by CK2-mediated phosphorylation of Dnmt3a. Cell Rep 8: 743-753, 2014.

4. Tsai LH, Wu JY, Cheng YW, Chen CY, Sheu GT, Wu TC and Lee H: The MZF1/c-MYC axis mediates lung adenocarcinoma progression caused by wild-type lkb1 loss. Oncogene 34: $1641-1649,2015$. 
5. Shen Y, Pan X and Zhao H: The histone demethylase PHF8 is an oncogenic protein in human non-small cell lung cancer. Biochem Biophys Res Commun 451: 119-125, 2014.

6. Wang C,Lu S, Jiang J, Jia X, Dong X and Bu P: Hsa-microRNA-101 suppresses migration and invasion by targeting Racl in thyroid cancer cells. Oncol Lett 8: 1815-1821, 2014.

7. Yan F, Shen N, Pang J, Xie D, Deng B, Molina JR, Yang P and Liu S: Restoration of miR-101 suppresses lung tumorigenesis through inhibition of DNMT3a-dependent DNA methylation. Cell Death Dis 5: e1413, 2014.

8. Zhu Y, Yu J, Wang S, Lu R, Wu J and Jiang B: Overexpression of CD133 enhances chemoresistance to 5-fluorouracil by activating the PI3K/Akt/p70S6K pathway in gastric cancer cells. Oncol Rep 32: 2437-2444, 2014.

9. Li J, Hart RP, Mallimo EM, Swerdel MR, Kusnecov AW and Herrup K: EZH2-mediated H3K27 trimethylation mediates neurodegeneration in ataxia-telangiectasia. Nat Neurosci 16 1745-1753, 2013.

10. Yan S, Jane DT, Dufresne MJ and Sloane BF: Transcription of cathepsin B in glioma cells: Regulation by an E-box adjacent to the transcription initiation site. Biol Chem 384: 1421-1427, 2003.

11. Yin J, Wang M, Jin C and Qi Q: miR-101 sensitizes A549 NSCLC cell line to CDDP by activating caspase 3-dependent apoptosis. Oncol Lett 7: 461-465, 2014.

12. Singh SK, Hawkins C, Clarke ID, Squire JA, Bayani J, Hide T, Henkelman RM, Cusimano MD and Dirks PB: Identification of human brain tumour initiating cells. Nature 432: 396-401, 2004.

13. Livak KJ and Schmittgen TD: Analysis of relative gene expression data using real-time quantitative PCR and the 2(-Delta Delta C(T)) Method. Methods 25: 402-408, 2001.

14. Yan S and Sloane BF: Molecular regulation of human cathepsin B: Implication in pathologies. Biol Chem 384: 845-854, 2003.

15. Sdek P, Oyama K, Angelis E, Chan SS, Schenke-Layland K and MacLellan WR: Epigenetic regulation of myogenic gene expression by heterochromatin protein 1 alpha. PLoS One 8: e58319, 2013.

16. Wang Z, Humphries B, Xiao H, Jiang Y and Yang C: MicroRNA-200b suppresses arsenic-transformed cell migration by targeting protein kinase $\mathrm{C} \alpha$ and Wnt5b-protein kinase $\mathrm{Co}$ positive feedback loop and inhibiting Racl activation. J Biol Chem 289: 18373-18386, 2014
17. Fang S, Zeng X, Zhu W, Tang R, Chao $\mathrm{Y}$ and Guo L: Zinc finger E-box-binding homeobox 2 (ZEB2) regulated by miR-200b contributes to multi-drug resistance of small cell lung cancer. Exp Mol Pathol 96: 438-444, 2014.

18. Men D, Liang Y and Chen L: Decreased expression of microRNA-200b is an independent unfavorable prognostic factor for glioma patients. Cancer Epidemiol 38: 152-156, 2014.

19. Liu Q, Tang H, Liu X, Liao Y, Li H, Zhao Z, Yuan X and Jiang W: miR-200b as a prognostic factor targets multiple members of RAB family in glioma. Med Oncol 31: 859, 2014.

20. Holmberg Olausson K, Maire CL, Haidar S, Ling J, Learner E, Nister M and Ligon KL: Prominin-1 (CD133) defines both stem and non-stem cell populations in CNS development and gliomas. PLoS One 9: e106694, 2014.

21. Park EY, Chang E, Lee EJ, Lee HW, Kang HG, Chun KH, Woo YM, Kong HK, Ko JY, Suzuki H, et al: Targeting of miR-34a-NOTCH1 axis reduced breast cancer stemness and chemoresistance. Cancer Res 74: 7573-7582, 2014.

22. Ren D, Wang M, Guo W, Huang S, Wang Z, Zhao X, Du H, Song L and Peng X: Double-negative feedback loop between ZEB2 and miR-145 regulates epithelial-mesenchymal transition and stem cell properties in prostate cancer cells. Cell Tissue Res 358: 763-778, 2014

23. Zhang D, Yang G, Chen X, Li C, Wang L, Liu Y, Han D, Liu H, Hou X, Zhang W, et al: mir-300 promotes self-renewal and inhibits the differentiation of glioma stem-like cells. J Mol Neurosci 53: 637-644, 2014.

24. Xiao Y, Wang J, Chen Y, Zhou K, Wen J, Wang Y, Zhou Y, Pan W and Cai W: Up-regulation of miR-200b in biliary atresia patients accelerates proliferation and migration of hepatic stallate cells by activating PI3K/Akt signaling. Cell Signal 26: 925-932, 2014.

25. Park JT, Kato M, Yuan H, Castro N, Lanting L, Wang M and Natarajan R: FOG2 protein down-regulation by transforming growth factor- $\beta 1$-induced microRNA-200b/c leads to Akt kinase activation and glomerular mesangial hypertrophy related to diabetic nephropathy. J Biol Chem 288: 22469-22480, 2013 